\title{
Inefficiency of Sera from Mice Treated with Pseudotyped SARS-CoV to Neutralize 2019-nCoV Infection
}

\author{
Zezhong Liu ${ }^{1} \cdot$ Shuai Xia ${ }^{1} \cdot$ Xinling Wang ${ }^{1} \cdot$ Qiaoshuai Lan $^{1} \cdot$ Wei X $\mathbf{u}^{1} \cdot$ Qian Wang $^{1} \cdot$ Shibo Jiang ${ }^{1}$ (D) \\ Lu Lu' ${ }^{1}$ (i)
}

Received: 22 February 2020 / Accepted: 14 March 2020/Published online: 31 March 2020

(c) Wuhan Institute of Virology, CAS 2020

Dear Editor,

An outbreak of unusual pneumonia in Wuhan, China recently was caused by infection of a novel type of coronavirus. The virus and disease were denoted as 2019-nCoV and COVID-19, respectively, by the World Health Organization (WHO). Most recently, 2019-nCoV was renamed SARS-CoV-2 by Coronaviridae Study Group (CSG) of the International Committee on Taxonomy of Viruses (ICTV) (Gorbalenya et al. 2020), or HCoV-19, as a common name for the consistence with COVID-19, by a group of virologists in China (Jiang et al. 2020a, b). As of 7 March 2020, a total of 80,651 confirmed cases, including 3070 deaths, were reported in China (China CDC 2020). Global spread is undeniable with serious implications for public health, thus calling for rapid development of effective therapeutics and prophylatics (Jiang et al. 2020a, b).

Very recently, it was found that horse anti-SARS-CoV and convalescent SARS patient sera had neutralizing effects against 2019-nCoV at a low dilution of 1:80 and 1:25, respectively (Zhou et al. 2020; Hoffmann et al. 2020). Although several SARS-CoV receptor-binding domain (RBD)-specific human monoclonal antibodies (mAbs), such as S230, m396 and 80R, exhibited no crossreactivity with 2019-nCoV RBD (Wrapp et al. 2020). CR3022, a SARS-CoV RBD-specific human mAb, was

Zezhong Liu and Shuai Xia have contributed equally to this work.

Electronic supplementary material The online version of this article (https://doi.org/10.1007/s12250-020-00214-5) contains supplementary material, which is available to authorized users.

Shibo Jiang

shibojiang@fudan.edu.cn

$凶 \mathrm{Lu} \mathrm{Lu}$

lul@fudan.edu.cn

1 Key Laboratory of Medical Molecular Virology (MOE/NHC/ CAMS), School of Basic Medical Sciences, Fudan University, Shanghai 200032, China reported to bind with the RBD of spike (S) protein in 2019-nCoV with potential cross-neutralizing activity (Tian et al. 2020). These studies suggest that the anti-SARS-CoV antibodies might be useful for the treatment of 2019-nCoV infection and people with history of SARS-CoV infection many years ago might be resistant to $2019-\mathrm{nCoV}$ infection.

The $\mathrm{S}$ protein in coronavirus plays an essential role in virus entry, and it is also the main target of neutralizing antibodies (Du et al. 2009). Sequence alignment indicated a $75.9 \%$ amino acid sequence identity between the $\mathrm{S}$ protein of SARS-CoV and that of 2019-nCoV (Supplementary Figure S1, Supplementary Table S1). SARS-related coronavirus (SARSr-CoV) WIV1 and Rs3367 have 92.3\% amino acid sequence identity to SARS-CoV in S protein, while those between SARS-CoV and MERS-CoV is $28.7 \%$ (Supplementary Figure S1, Supplementary Table S1). It is worth noting that the RBDs of SARS-CoV and 2019-nCoV exhibited a significant difference, although they bind the same receptor (angiotensin-converting enzyme 2, ACE2) during virus infection (Zhou et al. 2020). Therefore, despite sequence and receptor similarities, it is unclear whether the anti-SARS-CoV serum produced by immunization with $S$ protein-based vaccine could cross-neutralize 2019-nCoV infection.

We previously developed SARS pseudovirus (SARSPsV) and MERS pseudovirus (MERS-PsV), which were prepared by cotransfecting the plasmids of pNL4-3.luc.RE and pcDNA3.1-SARS, or MERS-CoV-S protein, to HEK293T cells (Fig. 1A) (Xia et al. 2019). These pseudoviruses (PsVs) bear the S protein and a defective HIV-1 genome, including a luciferase reporter gene, and could simulate the virus to infect target cells, thus having native conformations (Fig. 1A) (Xia et al. 2019). Consequently, we believed these PsVs to be potential immunogens to produce neutralizing antibodies. Female Balb/C mice $(\mathrm{n}=5)$ were subcutaneously injected with SARS-PsV, MERS-PsV or PBS as a control using Freund's complete adjuvant for the prime immunization and vaccinated at 
A

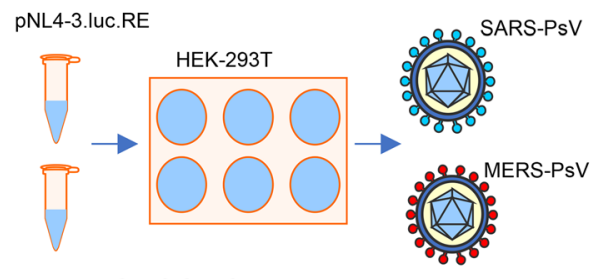

pCDNA3.1-SARS-CoV-S or pCDNA3.1-MERS-CoV-S

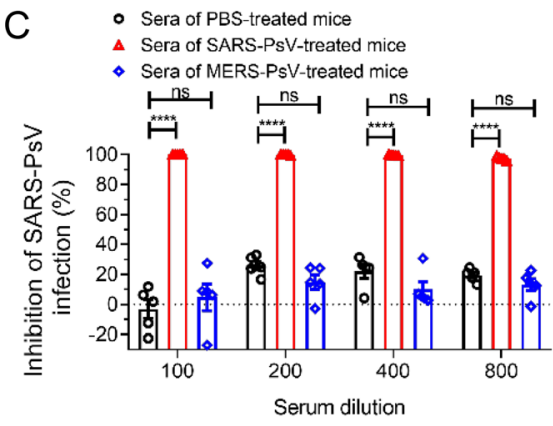

F
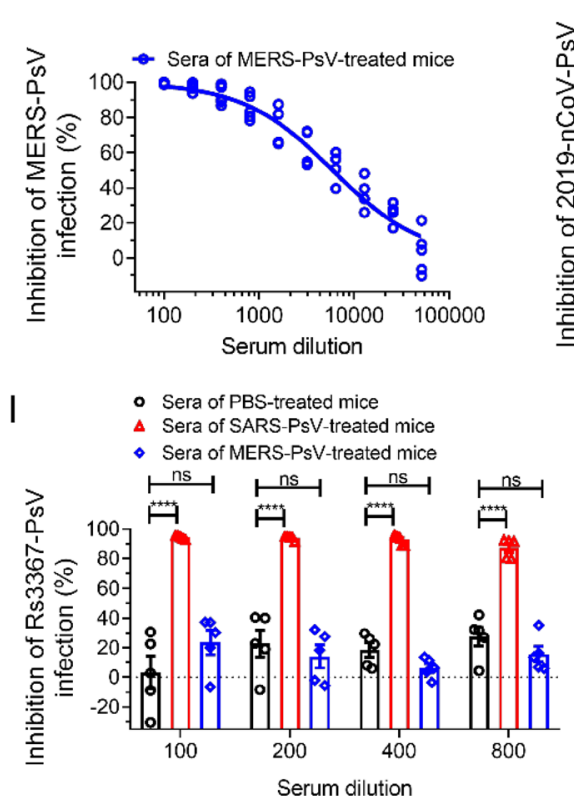

$\mathrm{L}$

\begin{tabular}{|l|l|l|l|l|l|l|l|l|l|l|l|l|l|l|l|}
\hline & $\begin{array}{l}\mathrm{i}-1 \\
\mathrm{NT}_{50}\end{array}$ & $\begin{array}{l}\mathrm{i}-2 \\
\mathrm{NT}_{50}\end{array}$ & $\begin{array}{l}\mathrm{i}-3 \\
\mathrm{NT}_{50}\end{array}$ & $\begin{array}{l}\mathrm{i}-4 \\
\mathrm{NT}_{50}\end{array}$ & $\begin{array}{l}\mathrm{i}-5 \\
\mathrm{NT}_{50}\end{array}$ & $\begin{array}{l}\mathrm{ii}-1 \\
\mathrm{NT}_{50}\end{array}$ & $\begin{array}{l}\mathrm{ii}-2 \\
\mathrm{NT}_{50}\end{array}$ & $\begin{array}{l}\mathrm{ii}-3 \\
\mathrm{NT}_{50}\end{array}$ & $\begin{array}{l}\mathrm{ii}-4 \\
\mathrm{NT}_{50}\end{array}$ & $\begin{array}{l}\mathrm{ii}-5 \\
\mathrm{NT}_{50}\end{array}$ & $\begin{array}{l}\mathrm{iii}-1 \\
\mathrm{NT}_{50}\end{array}$ & $\begin{array}{l}\text { iii-2 } \\
\text { NT }_{50}\end{array}$ & $\begin{array}{l}\text { iii-3 } \\
\text { NT }_{50}\end{array}$ & $\begin{array}{l}\text { iii-4 } \\
\text { NT }_{50}\end{array}$ & $\begin{array}{l}\text { iii-5 } \\
\text { NT }_{50}\end{array}$ \\
\hline SARS-PsV & 24319 & 57703 & 53705 & 42372 & 35248 & $<100$ & $<100$ & $<100$ & $<100$ & $<100$ & $<100$ & $<100$ & $<100$ & $<100$ & $<100$ \\
\hline WIV1-PsV & 7077 & 11456 & 9469 & 7299 & 10568 & $<100$ & $<100$ & $<100$ & $<100$ & $<100$ & $<100$ & $<100$ & $<100$ & $<100$ & $<100$ \\
\hline Rs3367-PsV & 5927 & 10185 & 8183 & 10055 & 4372 & $<100$ & $<100$ & $<100$ & $<100$ & $<100$ & $<100$ & $<100$ & $<100$ & $<100$ & $<100$ \\
\hline 2019-nCoV-PsV & $<100$ & $<100$ & $<100$ & $<100$ & $<100$ & $<100$ & $<100$ & $<100$ & $<100$ & $<100$ & $<100$ & $<100$ & $<100$ & $<100$ & $<100$ \\
\hline MERS-PsV & $<100$ & $<100$ & $<100$ & $<100$ & $<100$ & 4490 & 4175 & 9009 & 4456 & 9363 & $<100$ & $<100$ & $<100$ & $<100$ & $<100$ \\
\hline
\end{tabular}

B

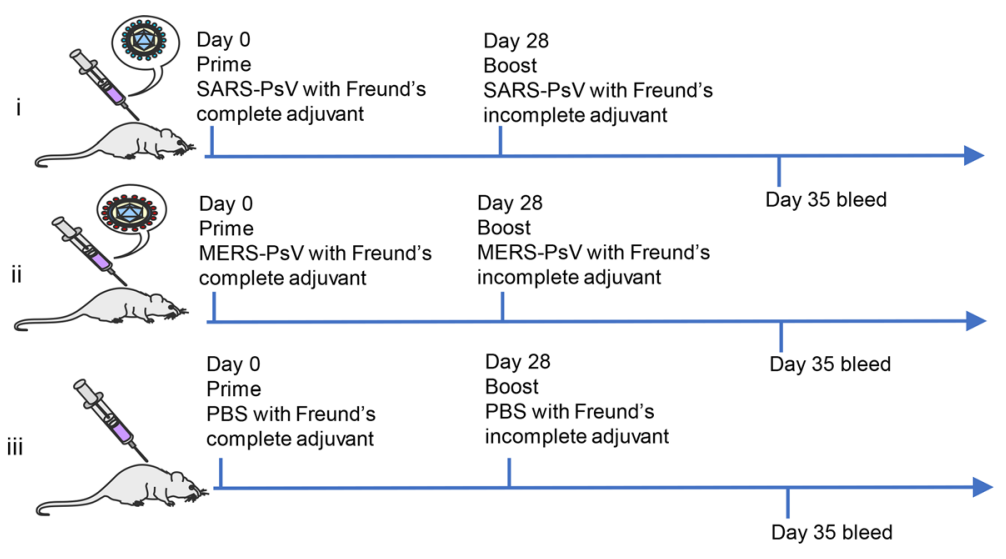

D

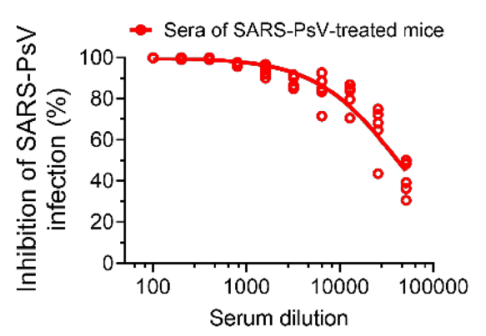

E

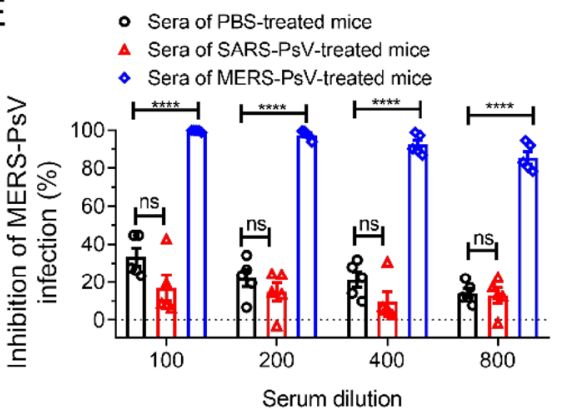

$\mathrm{H}$

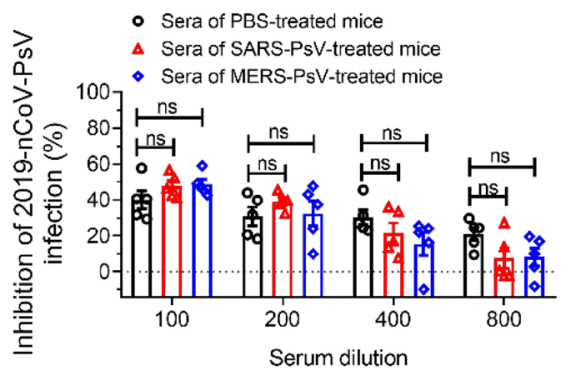

J

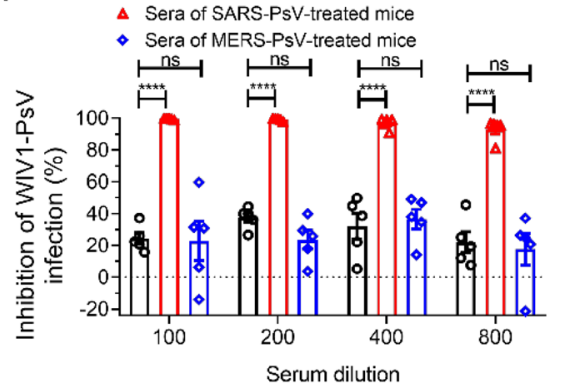

K

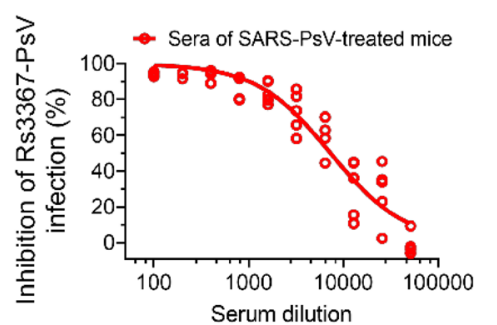


4Fig. 1 Neutralizing effects of sera from mice treated with SARS-PsV or MERS-PsV against SARS-PsV, MERS-PsV, 2019-nCoV-PsV, WIV1-PsV and Rs3367-PsV infection. A Schematic representation of the production of SARS-PsV and MERS-PsV. B The immunization procedure. C Detection of sera from SARS-PsV-, MERS-PsV- and PBS-treated mice for neutralization activity against SARS-PsV infection. D Assessment of sera from SARS-PsV-treated mice for neutralizing antibody titers against SARS-PsV infection. E Detection of sera from SARS-PsV-, MERS-PsV- and PBS-treated mice for neutralizing antibody titers against MERS-PsV infection. F Assessment of sera from MERS-PsV-treated mice for the neutralizing antibody titers against MERS-PsV infection. G Detection of sera from SARS-PsV-, MERS-PsV- and PBS-treated mice for neutralization activity against 2019-nCoV-PsV infection. H-I Detection of sera from SARS-PsV-, MERS-PsV- and PBS-treated mice for neutralization activity against WIV1-PsV and Rs3367-PsV, respectively. J$\mathbf{K}$ Detection of sera from SARS-PsV-treated mice for neutralization activity against WIV1-PsV and Rs3367-PsV infection. I NT No $_{50}$ value of mouse sera agaisnt SARS-PsV, MERS-PsV, WIV1-PsV, Rs3367PsV and 2019-nCoV-PsV infection. GraphPad Prism version 5.0 was used to perform all statistical analyses. ns, not significant; $* P<0.05$; $* * P<0.01 ; * * * P<0.001 ; * * * * P<0.0001$.

28 days after the first immunization using Freund's incomplete adjuvant for the boost immunization (Fig. 1B). Sera samples were collected from day 7 after the final vaccination.

We first tested day-35 sera for neutralization of SARSPsV infection using ACE2-expressing 293T cells. As shown in Fig. 1C, compared with the PBS-treated group, significant neutralization $(P<0.0001)$ was observed in the sera from SARS-PsV-treated mice at dilution of 1:100, 1:200, 1:400 and 1:800. The percent of inhibition of SARS$\mathrm{PsV}$ infection in sera from the SARS-PsV-vaccinated group could reach $\sim 97 \%$, even at a dilution of 1:800. Then, the $\mathrm{NT}_{50} \mathrm{~s}$ (50\% neutralizing antibody titers) of these sera were further tested. As shown in Fig. 1D, the mean $\mathrm{NT}_{50}$ of the sera was 41,425 . The sera from MERS-PsVtreated mice, as negative control, did not reach our $\mathrm{NT}_{50}$ threshold at a dilution of 1:100 and did not show a significant difference $(P>0.5)$ compared with the PBStreated control group. Then, we tested the neutralization effects of the sera against MERS-PsV infection using the Huh-7 cell line. We found significant MERS-PSV neutralization effects in the sera from MERS-PsV-treated mice (Fig. 1E). The $\mathrm{NT}_{50}$ of these sera was also detected as 5945 (Fig. 1F). However, the sera from SARS-PsV-treated mice could not significantly inhibit MERS-PsV infection, even at a dilution of 1:100 (Fig. 1E). In spite of the high neutralization titer in both groups, we did not observe a significant cross-neutralization effect.

To assess whether the SARS-PsV-treated mouse sera could show cross-neutralization effects for 2019-nCoV, we tested the inhibition effects of all sera using a 2019-nCoV pseudovirus system and Huh-7 cells as the target cell. No neutralization was detected, either for sera from SARS-PsV-treated mice or for MERS-PsV-treated mice, even at a dilution of 1: 100, compared with the sera from PBS-treated mice (Fig. 1G).

To investigate the ability to neutralize SARSr-CoVs, we further tested the inhibitory activities against two SARSrCoVs, WIV1 and Rs3367. Interestingly, sera from SARStreated mice could significantly neutralize WIVI-PsV and Rs3367-PsV with $\sim 92 \%$ and $\sim 87 \%$ inhibitory activities at a dilution of 1:800 (Fig. 1H and 1I). However, no neutralization was observed for MERS-PSV-treated mice sera, even at a dilution of 1:100, compared with the sera from PBS-treated control (Fig. 1H and 1I). Then, we further investigated whether the sera from SARS-CoV spike protein-treated mice could also cross-neutralize bat SARSrCoV, WIV1-PsV and Rs3367-PsV with high titers. The $\mathrm{NT}_{50}$ s for WIV1-PsV and Rs3367-PsV were 8787 and 7345, respectively (Fig. $1 \mathrm{~J}$ and $1 \mathrm{~K}$ ), demonstrating that sera from mice treated with SARS-CoV $\mathrm{S}$ protein could potently cross-neutralize infection by SARS-CoV and SARSr-CoVs, but weakly for 2019-nCoV infection, although its S protein sequence is highly similar to that of SARS-CoV spike protein (Supplementary Figure S1, Supplementary Table S1).

Taken together, our results indicated that the sera of mice treated with pseudotyped SARS-CoV exhibited low titer of 2019-nCoV neutralization activity $(<1: 100)$, implying that it may not be practical to treat $2019-\mathrm{nCoV}$ infection with anti-SARS-CoV antibodies and that people with history of SARS-CoV infection many years ago may not be resistant to 2019-nCoV infection. However, we cannot exclude the possibility that the low titer of the crossneutralizing antibody is due to the low density of S protein on the surface of pseudovirus particles. Furthermore, modifying or reconstructing the SARS-CoV S protein may enhance the exposure of conformational neutralizing epitopes in RBD, in order to increase its immunogenicity for eliciting higher cross-neutralizing antibody responses against 2019-nCoV and emerging/reemerging coronaviruses in the future.

Acknowledgements We thank Dr. Lanying Du of the Lindsley F. Kimball Research Institute, New York Blood Center, for providing the plasmids of pcDNA3.1-SARS or MERS-CoV-S. This work was supported by the National Megaprojects of China for Major Infectious Diseases (2018ZX10301403).

\section{Compliance with Ethical Standards}

Conflict of interest The authors declare that they have no conflict of interest.

Animal and Human Rights The mice related experiments were carried in strict accordance with institutional regulations (approval number 20190221-070, approval date 21 February 2019). 


\section{References}

China CDC (2020) Tracking the epidemic. http://weekly.chinacdc.cn/ news/TrackingtheEpidemic.htm?from=timeline\#Beijing\% 20Municipality\%20Update. Accessed 7 Mar 2020

Du L, He Y, Zhou Y, Liu S, Zheng BJ, Jiang S (2009) The spike protein of SARS-CoV-a target for vaccine and therapeutic development. Nat Rev Microbiol 7:226-236

Gorbalenya AE, Baker SC, Baric RS, Groot RJ, Drosten C, Gulyaeva AA, Haagmans BL, Lauber C, Leontovich AM, Neuman BW, Penzar D, Perlman S, Poon LLM, Samborskiy D, Sidorov IA, Sola I, Ziebuhr J (2020) Severe acute respiratory syndromerelated coronavirus - the species and its viruses, a statement of the Coronavirus Study Group. BioRxiv. https://doi.org/10.1101/ 2020.02.07.937862

Hoffmann M, Kleine-Weber H, Schroeder S, Krüger N, Herrler T, Erichsen S, Schiergens TS, Herrler G, Wu NH, Nitsche A, Müller MA, Drosten C, Pöhlmann S (2020) SARS-CoV-2 cell entry depends on ACE2 and TMPRSS2 and is blocked by a clinically proven protease inhibitor. Cell. https://doi.org/10. 1016/j.cell.2020.02.052

Jiang S, Shi Z, Shu Y, Song J, Gao G, Tan W, Guo D (2020a) A distinct name is needed for the new coronavirus. Lancet. https:// doi.org/10.1016/S0140-6736(20)30419-0
Jiang S, Du L, Shi Z (2020b) An emerging coronavirus causing pneumonia outbreak in Wuhan, China: calling for developing therapeutic and prophylactic strategies. Emerg Microb Infect 9:275-277

Tian X, Li C, Huang A, Xia S, Lu S, Shi Z, Lu L, Jiang S, Yang Z, Wu Y, Ying T (2020) Potent binding of 2019 novel coronavirus spike protein by a SARS coronavirus-specific human monoclonal antibody. Emerg Microb Infect 9:382-385

Wrapp D, Wang N, Corbett KS, Goldsmith JA, Hsieh CL, Abiona O, Graham BS, McLellan JS (2020) Cryo-EM structure of the 2019-nCoV spike in the prefusion conformation. Science 367:1260-1263

Xia S, Yan L, Xu W, Agrawal AS, Algaissi A, Tseng CK, Wang Q, Du L, Tan W, Wilson IA, Jiang S, Yang B, Lu L (2019) A pancoronavirus fusion inhibitor targeting the HR1 domain of human coronavirus spike. Sci Adv 5:eaav4580

Zhou P, Yang X, Wang X, Hu B, Zhang L, Zhang W, Si HR, Zhu Y, Li B, Huang CL, Chen HD, Chen J, Luo Y, Guo H, Jiang RD, Liu MQ, Chen Y, Shen XR, Wang X, Zheng XS, Zhao K, Chen QJ, Deng F, Liu LL, Yan B, Zhan FX, Wang YY, Xiao GF, Shi ZL (2020) A pneumonia outbreak associated with a new coronavirus of probable bat origin. Nature. https://doi.org/10. 1038/s41586-020-2012-7 